PROCEEDINGS OF THE

AMERICAN MATHEMATICAL SOCIETY

Volume 138, Number 4, April 2010, Pages 1415-1420

S 0002-9939(09)10158-2

Article electronically published on November 3, 2009

\title{
EXTREME AND EXPOSED POINTS OF SPACES OF INTEGRAL POLYNOMIALS
}

\author{
CHRISTOPHER BOYD AND SILVIA LASSALLE
}

(Communicated by Nigel J. Kalton)

\begin{abstract}
We show that if $E$ is a real Banach space such that $E^{\prime}$ has the approximation property and such that $\ell_{1} \hookrightarrow \widehat{\bigotimes}_{n, s, \epsilon} E$, then the set of extreme points of the unit ball of $\mathcal{P}_{I}\left({ }^{n} E\right)$ is equal to $\left\{ \pm \phi^{n}: \phi \in E^{\prime},\|\phi\|=1\right\}$. Under the additional assumption that $E^{\prime}$ has a countable norming set, we see that the set of exposed points of the unit ball of $\mathcal{P}_{I}\left({ }^{n} E\right)$ is also equal to $\left\{ \pm \phi^{n}: \phi \in\right.$ $\left.E^{\prime},\|\phi\|=1\right\}$.
\end{abstract}

\section{INTRODUCTION}

The isometric study of Banach spaces tells us that there are certain subsets on the unit sphere of a Banach space which are fundamental in our understanding of the geometry of Banach spaces. Such sets include the set of extreme and exposed points, the set of weak ${ }^{*}$-exposed and strongly exposed points and the set of denting points. We denote these sets by $\operatorname{Ext}(E), \operatorname{Exp}(E), w^{*} \operatorname{Exp}(E), \operatorname{sExp}(E)$, and $\operatorname{Dent}(E)$ respectively. These sets are used in the definition of such concepts as smoothness and strict convexity and in our understanding of concepts such as Fréchet differentiability and Gâteaux differentiability.

In the 1980's Ruess and Stegall published a series of papers where the geometric structure of duals of spaces of linear operators and of projective tensor products is investigated. In particular, given Banach spaces $E$ and $F$ such that either $E^{\prime}$ or $F^{\prime}$ has the approximation property and either $E^{\prime}$ or $F^{\prime}$ has the Radon-Nikodym property, it is shown in [1] that the extreme points of the unit ball of the projective tensor product of the duals, $E^{\prime} \widehat{\bigotimes}_{\pi} F^{\prime}$, is the set $\left\{x \otimes y: x \in \operatorname{Ext}\left(E^{\prime}\right), y \in \operatorname{Ext}\left(F^{\prime}\right)\right\}$. In 12 they prove that the analogous result holds for $\operatorname{Ext}\left(E^{\prime}\right)$ replaced with $\operatorname{Exp}\left(E^{\prime}\right)$, $w^{*} \operatorname{Exp}\left(E^{\prime}\right), \operatorname{sExp}\left(E^{\prime}\right)$ and $\operatorname{Dent}\left(E^{\prime}\right)$.

The theory of tensor products is also important in the study of spaces of homogeneous polynomials on Banach spaces. Here, however, it is the space of symmetric tensors which we need to investigate. The geometric structure of these spaces is very different to that of 'ordinary' tensor products.

Some definitions are in order. A point $x$ is said to be an extreme point of the (closed) unit ball of a Banach space $E, \bar{B}_{E}$, if $x$ cannot be written as the midpoint of a line segment which is entirely contained in $\bar{B}_{E}$. We recall that a unit vector $x$ in a Banach space $E$ is exposed if there is a unit vector $\phi \in E^{\prime}$ so that $\phi(x)=1$ and

Received by the editors February 3, 2009, and, in revised form, August 11, 2009.

2010 Mathematics Subject Classification. Primary 46G25; Secondary 46B04.

Key words and phrases. Integral polynomials, exposed points, extreme points.

(C)2009 American Mathematical Society 1415

Reverts to public domain 28 years from publication 
$\phi(y)<1$ for $y \in B_{E} \backslash\{x\}$. When $E=F^{\prime}$ is a dual space and $\phi$ is in $F$, we shall say that $x$ is weak*-exposed. A point $x$ in the unit ball of $E$ is said to be strongly exposed by $\phi$ in $S_{E^{\prime}}$ if whenever $\left(x_{k}\right)$ is a sequence in $E$ so that $\phi\left(x_{k}\right)$ converges to 1 , then $\left(x_{k}\right)$ converges to $x$ in norm.

Given a Banach space $E$, a point $x$ in the closed unit ball of $E$ is said to be a denting point if for every $\epsilon>0, x$ does not belong to $\overline{\Gamma\left(B_{E} \backslash B(x, \epsilon)\right)}$, the closure of the absolutely convex hull of $B_{E} \backslash B(x, \epsilon)$. This is equivalent to the condition that $x$ is contained in slices of the unit ball of $E$ which have arbitrary small diameter.

In [13. Ryan and Turett show that if $E$ is a real finite dimensional Banach space and $n>1$ is a positive integer, then the extreme points of the unit ball of $\widehat{\bigotimes}_{n, s, \pi} E$ are $\{ \pm x \otimes \cdots \otimes x: x \in E,\|x\|=1\}$. In $[3]$ an upper bound for the set of extreme points of the unit ball of $\mathcal{P}_{I}\left({ }^{n} E\right)$, the space of $n$-homogeneous integral polynomials on $E$, is given by $\left\{ \pm \phi^{n}: \phi \in E^{\prime},\|\phi\|=1\right\}$; see also [4. A lower bound is also obtained. Specifically it is shown in 3 that the set of extreme points of the unit ball $\mathcal{P}_{I}\left({ }^{n} E\right)$ contains $\left\{ \pm \phi^{n}: \phi \in E^{\prime},\|\phi\|=1\right.$ and $\phi$ attains its norm $\}$. Hence if $E$ is a real reflexive Banach space and $n$ is an integer which is greater than or equal to 2, then the set of extreme points of the unit ball of $P_{I}\left({ }^{n} E\right)$ is precisely the set $\left\{ \pm \phi^{n}: \phi \in E^{\prime},\|\phi\|=1\right\}$.

In this paper we show that given $E$ a real Banach space such that $E^{\prime}$ has the approximation property and fixed $n>1$ a positive integer such that $\widehat{\bigotimes}_{n, s, \epsilon} E$ does not contain a copy of $\ell_{1}$, then the set of extreme points of the unit ball of $\mathcal{P}_{I}\left({ }^{n} E\right)$ is $\left\{ \pm \phi^{n}: \phi \in E^{\prime},\|\phi\|=1\right\}$.

The extreme points of the unit ball of the space of $n$-homogeneous integral polynomials on complex Banach spaces are studied by Dineen in [8. For further reading on polynomials on infinite dimensional Banach spaces we refer the reader to 7 .

\section{EXtREME AND EXPOSED POINTS}

We begin by introducing some topologies on the space of symmetric tensor products and their relations with different spaces of polynomials. Given a tensor $u$ in $\bigotimes_{n, s} E$ we define the symmetric projective norm of $u$ indicated with $\pi$ to be

$$
\|u\|_{\pi}=\inf \left\{\sum_{i=1}^{k}\left\|x_{i}\right\|^{n}: u=\sum_{i=1}^{k} \lambda_{i} x_{i} \otimes \cdots \otimes x_{i}, \lambda_{i}= \pm 1, x_{i} \in E\right\} .
$$

We denote the completion of $\bigotimes_{n, s} E$ with respect to the projective symmetric norm by $\widehat{\bigotimes}_{n, s, \pi} E$. The dual of $\widehat{\bigotimes}_{n, s, \pi} E$ is the space $\mathcal{P}\left({ }^{n} E\right)$ of all continuous $n$-homogeneous polynomials on $E$ endowed with the norm $\|P\|=\sup _{\|x\| \leq 1}|P(x)|$.

An $n$-homogeneous polynomial $P$ on $E$ is said to be nuclear if there is a bounded sequence $\left(\phi_{j}\right)_{j=1}^{\infty} \subset E^{\prime}$ and a sequence $\left(\lambda_{j}\right)_{j=1}^{\infty}$ in $\ell_{1}$ such that

$$
P(x)=\sum_{j=1}^{\infty} \lambda_{j} \phi_{j}(x)^{n}
$$

for every $x$ in $E$. The space of all nuclear $n$-homogeneous polynomials on $E$ is denoted by $\mathcal{P}_{N}\left({ }^{n} E\right)$ and becomes a Banach space when the norm of $P$ is given as the infimum of $\sum_{j=1}^{\infty}\left|\lambda_{j}\right|\left\|\phi_{j}\right\|^{n}$ taken over all representations of $P$ of the form described above. This norm is called the nuclear norm of $P$ and is denoted by 
$\|P\|_{N}$. Given $\phi$ in $E^{\prime}$ we denote by $\phi^{n}$ the $n$-homogeneous polynomial which takes $x$ to $\phi(x)^{n}$. When $E^{\prime}$ has the approximation property, $\mathcal{P}_{N}\left({ }^{n} E\right)$ is isometrically isomorphic to $\widehat{\bigotimes}_{n, s, \pi} E^{\prime}$ under the map induced by $\phi^{n} \rightarrow \phi \otimes \cdots \otimes \phi$.

A polynomial $P$ on $E$ is said to be integral if there is a regular Borel measure $\mu$ on $\left(B_{E^{\prime}}, \sigma\left(E^{\prime}, E\right)\right)$ such that

$$
P(x)=\int_{B_{E^{\prime}}} \phi(x)^{n} d \mu(\phi)
$$

for every $x$ in $E$. We write $\mathcal{P}_{I}\left({ }^{n} E\right)$ for the space of all $n$-homogeneous integral polynomials on $E$. We define the integral norm of an integral polynomial $P,\|P\|_{I}$, as the infimum of $\|\mu\|$ taken over all regular Borel measures which satisfy (1). This space is the dual of the symmetric injective tensor product. Given an $n$-fold symmetric tensor $u=\sum_{i=1}^{k} \lambda_{i} x_{i} \otimes \cdots \otimes x_{i}$ on $E$, its symmetric injective norm indicated with $\epsilon$ is defined as

$$
\|u\|_{\epsilon}=\sup _{\phi \in B_{E^{\prime}}}\left|\sum_{i=1}^{k} \lambda_{i} \phi\left(x_{i}\right)^{n}\right| .
$$

We denote the completion of $\bigotimes_{n, s} E$ with respect to this norm by $\widehat{\bigotimes}_{n, s, \epsilon} E$. It follows from [6] that the dual of $\widehat{\bigotimes}_{n, s, \epsilon} E$ is isometrically isomorphic to $\left(\mathcal{P}_{I}\left({ }^{n} E\right),\|\cdot\|_{I}\right)$.

Given a Banach space $E$, the space of $n$-fold symmetric tensors $\bigotimes_{n, s} E$ may be considered as a subspace of $\widehat{\bigotimes}_{n, s, \pi} \ell_{\infty}\left(B_{E^{\prime}}\right)$. We let $\bigotimes_{n, s, \eta} E$ denote $\bigotimes_{n, s} E$ with the norm induced from $\widehat{\bigotimes}_{n, s, \pi} \ell_{\infty}\left(B_{E^{\prime}}\right)$. The completion of $\bigotimes_{n, s, \eta} E$ is denoted by $\widehat{\bigotimes}_{n, s, \eta} E$. The space $\ell_{\infty}\left(B_{E^{\prime}}\right)$ may be replaced by $C\left(B_{E^{\prime}}, w^{*}\right)$ in the construction of $\widehat{\bigotimes}_{n, s, \eta} E$. Since the projective tensor product does not respect subspaces, there is no Hahn-Banach Theorem for $n$-homogeneous polynomials when $n$ is at least 2. In [10] Kirwan and Ryan introduce the space of all extendible $n$-homogeneous polynomials as those polynomials which extend continuously to every superspace, and they show that this subclass is isometrically isomorphic to the dual of $\widehat{\bigotimes}_{n, s, \eta} E$.

Although the class of integral polynomials is embedded in the class of extendible polynomials, there are extendible nonintegral polynomials; for example $c_{0}$ contains nonintegral extendible polynomials (see [5]). However, we shall see that the study of the geometry of $\widehat{\bigotimes}_{n, s, \eta} E$ reveals information about the geometry of the spaces of integral polynomials and $n$-fold symmetric tensors.

Lemma 1. Let $E$ be a real Banach space and $n>1$ be a positive integer. Then $\left\{x^{n}:\|x\|=1\right\} \subseteq \operatorname{Ext}\left(\widehat{\bigotimes}_{s, n, \eta} E\right)$.

Proof. From the inclusion $\widehat{\bigotimes}_{s, n, \eta} E \hookrightarrow \widehat{\bigotimes}_{s, n, \pi} \ell_{\infty}\left(B_{E^{\prime}}\right)$ and the fact that $\operatorname{Ext}(B) \cap$ $A \subset \operatorname{Ext}(A)$ whenever $A \subset B$, we have that

$$
\operatorname{Ext}\left(\widehat{\otimes}_{s, n, \pi} \ell_{\infty}\left(B_{E^{\prime}}\right)\right) \cap \widehat{\otimes}_{s, n, \eta} E \subseteq \operatorname{Ext}\left(\widehat{\otimes}_{s, n, \eta} E\right) .
$$

On the other hand, as $\ell_{\infty}\left(B_{E^{\prime}}\right)$ has the approximation property $\widehat{\bigotimes}_{s, n, \pi} \ell_{\infty}\left(B_{E^{\prime}}\right)$ $=\mathcal{P}_{N}\left({ }^{n} \ell_{1}\left(B_{E^{\prime}}\right)\right)$, by [3, Proposition 1], the extreme points of the unit ball of $\mathcal{P}_{I}\left({ }^{n} \ell_{1}\left(B_{E^{\prime}}\right)\right)$ are all nuclear polynomials; therefore,

$$
\operatorname{Ext}\left(\mathcal{P}_{I}\left({ }^{n} \ell_{1}\left(B_{E^{\prime}}\right)\right)\right) \subseteq \operatorname{Ext}\left(\mathcal{P}_{N}\left({ }^{n} \ell_{1}\left(B_{E^{\prime}}\right)\right)\right)
$$


Applying [3. Proposition 5], we have that

$$
\begin{aligned}
\left\{ \pm \phi^{n}: \phi \in \ell_{\infty}\left(B_{E^{\prime}}\right) ;\|\phi\|=1 ; \phi \text { norm attaining }\right\} & \subseteq \operatorname{Ext}\left(\mathcal{P}_{I}\left({ }^{n} \ell_{1}\left(B_{E^{\prime}}\right)\right)\right) \\
& \subseteq \operatorname{Ext}\left(\widehat{\bigotimes}_{s, n, \pi} \ell_{\infty}\left(B_{E^{\prime}}\right)\right) .
\end{aligned}
$$

Use $\hat{x}$ to denote the canonical image of $x \in E$ in $\ell_{\infty}\left(B_{E^{\prime}}\right)$. Since $\hat{x}$ is norm attaining, $\left\{\hat{x}^{n}:\|x\|=1\right\} \subseteq \operatorname{Ext}\left(\widehat{\otimes}_{s, n, \pi} \ell_{\infty}\left(B_{E^{\prime}}\right)\right)$. Finally, $\left\{x^{n}:\|x\|=1\right\} \subseteq$ $\operatorname{Ext}\left(\widehat{\bigotimes}_{s, n, \pi} \ell_{\infty}\left(B_{E^{\prime}}\right)\right) \cap \widehat{\bigotimes}_{s, n, \eta} E$, and therefore $\left\{x^{n}:\|x\|=1\right\} \subseteq \operatorname{Ext}\left(\widehat{\bigotimes}_{s, n, \eta} E\right)$.

This result is the key to classifying the extreme points of the space of $n$-homogeneous integral polynomials when $\ell_{1}$ is not a subspace of $\widehat{\bigotimes}_{n, s, \epsilon} E$.

Theorem 2. Let $E$ be a real Banach space so that $E^{\prime}$ has the approximation property. Let $n>1$ be a positive integer and suppose that $\widehat{\bigotimes}_{n, s, \epsilon} E$ does not contain a subspace isomorphic to $\ell_{1}$. Then the set of extreme points of the unit ball of $\mathcal{P}_{I}\left({ }^{n} E\right)$ is equal to $\left\{ \pm \phi^{n}: \phi \in E^{\prime},\|\phi\|=1\right\}$.

Proof. Since $\ell_{1} \hookrightarrow \widehat{\bigotimes}_{n, s, \epsilon} E$ and $E^{\prime}$ has the approximation property, it follows from [3. Theorem 2] (or [4, Theorem 1.5]) that $\mathcal{P}_{I}\left({ }^{n} E\right)$ is isometrically isomorphic to $\widehat{\bigotimes}_{n, s, \pi} E^{\prime}$.

By [9, 18.3.7], the mapping $\widehat{\bigotimes}_{s, n, \pi} E^{\prime} \hookrightarrow \widehat{\bigotimes}_{s, n, \epsilon} E^{\prime}$ is injective whenever $E^{\prime}$ has the approximation property. Hence, the canonical mapping $j: \widehat{\bigotimes}_{s, n, \pi} E^{\prime} \hookrightarrow$ $\widehat{\bigotimes}_{s, n, \eta} E^{\prime}$ is also injective.

Let $\phi^{n}$ be in $\widehat{\bigotimes}_{s, n, \pi} E^{\prime}$ with $\|\phi\|=1$. Since $\left\|\phi^{n}\right\|_{\pi}=\left\|\phi^{n}\right\|_{\eta}=\|\phi\|^{n}$, then, by Lemma 1, $\phi^{n} \in \operatorname{Ext}\left(\widehat{\bigotimes}_{s, n, \eta} E^{\prime}\right)$.

In order to prove that $\phi^{n}$ is an extreme point of the unit ball of $\widehat{\bigotimes}_{s, n, \pi} E^{\prime}$, suppose that is not the case. Then we can find $u \in \widehat{\bigotimes}_{s, n, \pi} E^{\prime}, u \neq 0$ so that $\left\|\phi^{n} \pm u\right\|_{\pi} \leq 1$. Thus $\left\|j\left(\phi^{n} \pm u\right)\right\|_{\eta} \leq 1$ and $j(u)=0$ in $\widehat{\bigotimes}_{s, n, \eta} E^{\prime}$; this leads us into a contradiction since $j$ is injective.

The reverse inclusion is a consequence of the fact that $\mathcal{P}_{I}\left({ }^{n} E\right)=\widehat{\bigotimes}_{s, n, \pi} E^{\prime}$ together with [3, Proposition 1].

Since the injective tensor product of Asplund spaces is Asplund and Asplund spaces cannot contain a copy of $\ell_{1}$, we obtain the following corollary:

Corollary 3. Let $E$ be a real Banach space. Suppose that $E$ is Asplund and that $E^{\prime}$ has the approximation property. Then the set of extreme points of the unit ball of $\mathcal{P}_{I}\left({ }^{n} E\right)$ is equal to $\left\{ \pm \phi^{n}: \phi \in E^{\prime},\|\phi\|=1\right\}$, for any $n>1$ a positive integer.

In the course of proving Theorem 2, we have also proved the following result.

Corollary 4. Let $E$ be a real Banach space. Then, for any $n>1$ a positive integer, the set $\left\{ \pm x^{n}: x \in E,\|x\|=1\right\}$ is contained in the set of extreme points of the unit ball of $\widehat{\bigotimes}_{s, n, \pi} E$.

Let us now investigate the exposed points of the space of integral polynomials. 
Lemma 5. Let $E$ be a real Banach space such that $E^{\prime}$ has a countable norming set. Let $n>1$ be a positive integer. Then $\left\{x^{n}:\|x\|=1\right\} \subseteq \operatorname{Exp}\left(\widehat{\bigotimes}_{s, n, \eta} E\right)$.

Proof. Let $x$ be a point of $E$ with $\|x\|=1$. Choose $\phi$ in $E^{\prime}$ so that $\phi(x)=1$. Let $K$ be a countable norming subset of $E^{\prime}$ and define $K_{x}$ to be $K \cup\{\phi\}$. Applying an analogous argument to that given at the beginning of Lemma 1 , we see that

$$
\operatorname{Exp}\left(\widehat{\bigotimes}_{s, n, \pi} \ell_{\infty}\left(K_{x}\right)\right) \bigcap \widehat{\bigotimes}_{s, n, \eta} E \subseteq \operatorname{Exp}\left(\widehat{\bigotimes}_{s, n, \eta} E\right) .
$$

Since every exposed point of the unit ball of $\mathcal{P}_{I}\left({ }^{n} \ell_{1}\left(K_{x}\right)\right)$ is also an exposed point of the unit ball of $\mathcal{P}_{N}\left({ }^{n} \ell_{1}\left(K_{x}\right)\right)$, we get that

$$
\operatorname{Exp}\left(\mathcal{P}_{I}\left({ }^{n} \ell_{1}\left(K_{x}\right)\right)\right) \subseteq \operatorname{Exp}\left(\mathcal{P}_{N}\left({ }^{n} \ell_{1}\left(K_{x}\right)\right)\right)=\operatorname{Exp}\left(\widehat{\bigotimes}_{s, n, \pi} \ell_{\infty}\left(K_{x}\right)\right) .
$$

Using [3, Theorem 8] and the fact that weak*-exposed points are exposed points, we have

$$
\begin{aligned}
\left\{ \pm \phi^{n}: \phi \in \ell_{\infty}\left(K_{x}\right),\|\phi\|=1, \phi \text { is norm attaining }\right\} & \subseteq \operatorname{Exp}\left(\mathcal{P}_{I}\left({ }^{n} \ell_{1}\left(K_{x}\right)\right)\right) \\
& \subseteq \operatorname{Exp}\left(\widehat{\bigotimes}_{s, n, \pi} \ell_{\infty}\left(K_{x}\right)\right) .
\end{aligned}
$$

Consider $\hat{x}$, the image of $x$ in $\ell_{\infty}\left(K_{x}\right)$. Then $\hat{x}$ attains its norm at the point $\delta_{\phi}$ where

$$
\delta_{\phi}(\psi)= \begin{cases}1 & \text { if } \phi=\psi \\ 0 & \text { if } \phi \neq \psi\end{cases}
$$

Therefore we see that $\left\{\hat{x}^{n}:\|x\|=1\right\} \subseteq \operatorname{Exp}\left(\widehat{\bigotimes}_{s, n, \pi} \ell_{\infty}\left(K_{x}\right)\right)$. The remainder of the proof follows as in Lemma 1,

Theorem 6. Let $E$ be a real Banach space such that $E^{\prime}$ has the approximation property and a countable norming set. Let $n>1$ be a positive integer and suppose that $\widehat{\bigotimes}_{n, s, \epsilon} E$ does not contain a subspace isomorphic to $\ell_{1}$. Then the set of exposed points of the unit ball of $\mathcal{P}_{I}\left({ }^{n} E\right)$ is equal to $\left\{ \pm \phi^{n}: \phi \in E^{\prime},\|\phi\|=1\right\}$.

Proof. Since $\ell_{1}$ is not a subspace of $\widehat{\bigotimes}_{n, s, \epsilon} E$, it follows from [3, Theorem 2] or [4, Theorem 1.5] that $\mathcal{P}_{I}\left({ }^{n} E\right)$ is isometrically isomorphic to $\widehat{\bigotimes}_{s, n, \pi} E^{\prime}$. Since $\|\cdot\|_{\eta} \leq$ $\|\cdot\|_{\pi}$ each exposed point of the unit ball of $\widehat{\bigotimes}_{s, n, \eta} E^{\prime}$ is also an exposed point of the unit ball of $\widehat{\bigotimes}_{s, n, \pi} E^{\prime}$. (Simply restrict the exposing functional to $\widehat{\bigotimes}_{s, n, \pi} E^{\prime}$.) Thus the exposed points of the unit ball of $\mathcal{P}_{I}\left({ }^{n} E\right)$ contain $\left\{ \pm \phi^{n}: \phi \in E^{\prime},\|\phi\|=1\right\}$. The reverse inclusion follows immediately from [3, Proposition 5].

The above result applies to all real Asplund Banach spaces $E$ such that $E^{\prime}$ has the approximation property and a countable norming set. In particular, it holds for all real separable Asplund Banach spaces $E$ such that $E^{\prime}$ has the approximation property.

The methods used in this paper to give that the extreme and exposed points of the space of integral polynomials are, up to multiplication by \pm 1 , a power of a linear functional will not work for denting or strongly exposed points. To see this we note that both methods are based on embedding the space, of symmetric $n$-tensors of $E$ in the space of symmetric $n$-tensors of $\ell_{\infty}\left(B_{E^{\prime}}\right)$ or $\ell_{\infty}\left(K_{x}\right)$ for some countable norming subset $K_{x}$ of $E^{\prime}$. However, it is shown in [1, Proposition 2.2] (see also [2]) that if $K$ is an infinite compact Hausdorff topological space, then every slice of the 
unit ball of $\widehat{\bigotimes}_{n, s, \pi} C(K)$ has diameter 2. Hence the unit ball of $\widehat{\bigotimes}_{n, s, \pi} C(K)$ has no denting points and therefore no strongly exposed points. On the other hand 3 , Section 4] gives examples of Banach spaces $E$ where each $x^{n}$ is a strongly exposed point of $\widehat{\bigotimes}_{n, s, \pi} E$.

\section{ACKNOWLEDGEMENT}

The authors wish to thank the referee for a careful reading of the paper and for the suggestions made to improve it.

\section{REFERENCES}

[1] Acosta, M. and Becerra Guerrero, J., Slices in the unit ball of the symmetric tensor product of $\mathrm{C}(K)$ and $L_{1}(\mu)$, Ark. Mat., 47 (1) (2009), 1-12. MR.2480913

[2] Acosta, M. and Becerra Guerrero, J., Slices in the unit ball of the symmetric tensor product of a Banach space, J. Convex Anal., to appear.

[3] Boyd, C. and Ryan, R.A., Geometric theory of integral polynomials and symmetric tensor products, J. Functional Analysis, 179 (2001), 18-42. MR1807251 (2002b:46029)

[4] Carando, D. and Dimant, V., Duality in spaces of nuclear and integral polynomials, J. Math. Anal. Appl., 241 (2000), 107-121. MR.1738337 (2001c:46089)

[5] Carando, D. and Zalduendo, I., A Hahn-Banach theorem for integral polynomials, Proc. Amer. Math. Soc., 127 (1999), 241-250. MR.1458865 (99b:46067)

[6] Dineen, S., Holomorphy types on a Banach space, Studia Math., 39 (1971), 241-288. MR0304705 (46:3837)

[7] Dineen, S., Complex analysis on infinite-dimensional spaces, Monographs in Mathematics, Springer-Verlag, London, 1999. MR1705327 (2001a:46043)

[8] Dineen, S., Extreme integral polynomials on a complex Banach space, Math. Scand., 92 (2003), 129-140. MR.1951449 (2003j:46064)

[9] Jarchow, H., Locally convex spaces, B.G. Teubner, Stuttgart, 1981. MR632257 (83h:46008)

[10] Kirwan, P. and Ryan, R.A., Extendibility of homogeneous polynomials on Banach spaces, Proc. Amer. Math. Soc., 126 (1998), 1023-1029. MR.1415346 (98f:46042)

[11] Ruess, W.M. and Stegall, C.P., Extreme points in duals of operator spaces, Math. Ann., 261 (1982), 535-546. MR682665 (84e:46007)

[12] Ruess, W.M. and Stegall, C.P., Exposed and denting points in duals of operator spaces, Israel J. Math., 53 (1986), 163-190. MR845870 (87j:46015)

[13] Ryan, R.A. and Turett, B., Geometry of spaces of polynomials, J. Math. Anal. Appl., 221 (1998), 698-711. MR1621703 (99g:46015)

School of Mathematical Sciences, University College Dublin, Belfield, Dublin 4, IRELAND

E-mail address: Christopher.Boyd@ucd.ie

Departamento de Matemática, Pab. I - Cuidad Universitaria (FCEN), Universidad de Buenos Aires, (1428) Buenos Aires, Argentina

E-mail address: slassall@dm.uba.ar 